\title{
Efficacy and safety of iron supplementation in patients with heart failure and iron deficiency: a meta-analysis
}

\author{
Shuo Zhang ${ }^{1} \dagger$, Fengxiao Zhang ${ }^{2} \dagger$, Meng $\mathrm{Du}^{2}$, Kun Huang ${ }^{2}$ and Cheng Wang ${ }^{2 *}$ \\ ${ }^{1}$ First Clinical College, Union Hospital, Tongji Medical College, Huazhong University of Science and Technology, Wuhan, \\ 430022, People's Republic of China \\ ${ }^{2}$ Department of Cardiology, Union Hospital, Tongji Medical College, Huazhong University of Science and Technology, Wuban, \\ 430022, People's Republic of China
}

(Submitted 16 April 2018 - Final revision received 7 December 2018 - Accepted 8 January 2019)

\section{Abstract}

Fe therapy can be effective in heart failure patients both with and without anaemia. However, the role of Fe therapy in such patients is still uncertain. In this review, the aim was to evaluate the efficacy and safety of Fe therapy in adult patients with heart failure who have reduced ejection fraction (HFrEF). Multiple databases (PubMed, Medline, EMBASE, the Cochrane Library and Clinical Trials) were searched up to December 2017 and the reference lists of relevant articles obtained from the search were reviewed. Data extracted from randomised control trials (RCT) selected for the review were pooled using a fixed effects model or a random effects model, according to heterogeneity between trials. Nine RCT were included in this meta-analysis which included a total of 789 patients who received Fe therapy and who in turn were compared with 585 controls. There was significant improvement in the 6-min walk test $(19 \cdot 05 \mathrm{~m}, 95 \% \mathrm{CI} 10 \cdot 48,27 \cdot 62)$ and peak VO$/ 2 \mathrm{~kg}$ $(0.93 \mathrm{ml} / \mathrm{kg}$ per $\min , 95 \% \mathrm{CI} 0 \cdot 16,1.69)$ in the Fe supplementation arm. With Fe therapy, fewer patients were hospitalised for heart failure (OR: $0 \cdot 42,95 \%$ CI $0 \cdot 27,0 \cdot 65$ ), but no relationship was found for total re-hospitalisation (OR: $0 \cdot 70,95 \%$ CI $0 \cdot 32,1 \cdot 51$ ) or mortality (OR: $0 \cdot 70,95 \%$ CI $0 \cdot 38,1 \cdot 28)$. Fe therapy has the potential to improve exercise tolerance, reduce re-hospitalisations for patients with HFrEF having Fe deficiency. In addition, Fe supplementation was found to be safe, with no increased rate of adverse events.

Key words: Heart failure: Iron: Iron deficiency: Meta-analyses

Heart failure (HF) is well documented as a global health problem not only because it is a cause of increased morbidity and mortality, but also because it is responsible for high socio-economic costs ${ }^{(1,2)}$. An epidemiological investigation in 2000 demonstrated that the morbidity due to HF was $0.9 \%$ in China ${ }^{(3)}$, and one in nine deaths in America was found to be related to $\mathrm{HF}^{(4)}$. Despite advances in the moderation of mortality, hospitalisation for $\mathrm{HF}$ is still frequent and re-admission rates continue to rise ${ }^{(5)}$.

Fe performs a crucial role in oxygen transport and storage because it is a key component of $\mathrm{Hb}^{(6,7)}$. Until recently, $\mathrm{Fe}$ deficiency (ID) was ignored despite it being a common and stand-alone co-morbidity in $\mathrm{HF}$ associated with poor physical activity and adverse outcome, independent of anaemia status ${ }^{(8-10)}$. ID, the most common cause of anaemia, is becoming increasingly prevalent in HF patients, which is worth investigation and further discussion.

Recently, it has been recognised that the effect of anaemia treatment on $\mathrm{HF}$ patients using erythropoietin and $\mathrm{Fe}$ supplementation significantly improves cardiac function and quality of life $(\mathrm{QOL})^{(8)}$. A randomised controlled trial (RCT) carried out by Silverberg et al. ${ }^{(11)}$ confirmed that erythropoietin and intravenous Fe treatment significantly enhanced cardiac function and reduced hospitalisation but without apparent increase in adverse events. Bolger et al. ${ }^{(12)}$ found in an observational study that intravenous Fe sucrose alone is a simple and safe therapy that increases the capacity for exercise and relieves symptoms in chronic HF patients.

However, the data produced in these studies were too underpowered to establish that $\mathrm{Fe}$ therapy provides clinical benefit in patients with heart failure who have reduced ejection fraction (HFrEF) having ID. Many further clinical trials have since been conducted, which claim to provide more detailed analysis to obtain a more accurate conclusion. Therefore, we carried out a meta-analysis to summarise the evidence from these RCT to evaluate the safety and efficacy of Fe therapy in patients with HFrEF having ID.

Abbreviations: 6MWT, 6-min walk test; HF, heart failure; HFrEF, heart failure with reduced ejection fraction; ID, Fe deficiency; RCT, randomised control trial; TSAT, transferrin saturation.

* Corresponding author: C. Wang, fax +27 87679477, email wangchengv@hust.edu.cn

$\dagger$ Dr Shuo Zhang and Fengxiao Zhang contributed equally. 


\section{Methods}

\section{Search strategy}

We performed a systematic literature search of PubMed, MEDLINE, EMBASE and the Cochrane Library databases, in addition to conference proceedings and the Clinical Trials database for ongoing and unpublished trials. The reference lists of all articles obtained through the search process were reviewed for additional trials. Specifically, the search query was "("heart failure" OR "cardiac failure" OR "myocardial failure" OR "heart decompensation") AND ("ferric" OR "ferrous" OR "iron")'. The search was restricted to articles about humans and published up to December 2017.

\section{Inclusion and exclusion criteria}

The search results were limited to RCT. The inclusion criteria were as follows: (1) symptomatic stages of congestive heart failure (CHF) (New York Heart Association class II-IV) with ejection fraction (EF) lower than 45\%; (2) ID: serum ferritin lower than $100 \mathrm{ng} / \mathrm{ml}$ or serum ferritin between 100 and $299 \mathrm{ng} / \mathrm{ml}$ with transferrin saturation (TSAT) $<20 \%$; (3) intervention: oral or intravenous Fe preparations and (4) control group: placebo or no treatment. Trials were excluded if they included patients under 18, pregnant women or patients with active bleeding. Trials that were still recruiting patients or without any available quantifiable data were excluded. There were no restrictions on publication status (published, conference proceedings or unpublished), publication year or language. Research results were independently screened by two reviewers (C. W. and S. Z.) using a structured literature tool (Endnote X7; Thomson Reuters). Any disagreements were resolved through consensus reached by discussion with a third researcher (F. Z.).

\section{Data extraction and quality assessment}

Two reviewers (C. W. and S. Z.) independently extracted the following data from the trials included in the review: first author's family name, year of publication, number of participants, baselines, clinical end points and adverse events. Disagreements were resolved through discussion with a third researcher (F. Z).

The trials were assessed by examination of the following aspects: random sequence generation, allocation concealment, blinding of subjects and participants, blinding of outcome assessment and statistical analysis, integrity of follow-up visit, incomplete or selective outcome data reporting and baseline comparability. Each domain was graded as low risk, unclear risk (where there was lack of information or uncertainty over the potential for bias) or high risk (for bias according to the criteria specified in the Cochrane Handbook version 5.1.0. $)^{(13,14)}$.

\section{Definition of outcomes}

The primary outcome measures were peak $\mathrm{VO}_{2} / \mathrm{kg}$ and change in distance for the $6 \mathrm{~min}$ walk test (6MWT) over baseline measurement.

Secondary outcomes included mortality, number of hospitalisations during follow-up, adverse effects related to treatment,
Minnesota Living with Heart Failure Questionnaire (MLHFQ), European Quality of Life-5 Dimensions (EQ-5D) and Fe metabolism biomarkers, such as Hb levels, serum ferritin and TSAT.

\section{Statistical analysis}

Data analysis was performed using RevMan 5.3 (The Cochrane Collaboration). Dichotomous data were analysed by calculating the OR for each trial, with $95 \%$ CI for the uncertainty of each result. Mean and standard deviation values were obtained for continuous variables, including calculating a weighted mean difference for variables on the same scale from different trials. A fixed effects model was used throughout the review, except in the event of significant heterogeneity between trials $\left(I^{2}>50 \%\right)$, in which case a random effects model was used in the analysis. Mean and variance from the median, range and size of sample were estimated when the mean and variance were not directly acquired. Publication bias was not assessed because of the limited number (below 10) of studies selected ${ }^{(13,15)}$.

\section{Results}

\section{Search results and study characteristics}

From the initial search, 3527 articles and abstracts (including duplicates) were extracted, with an additional six from other sources that were identified and retrieved for further review. The evaluation excluded 2898 of these, with seventy-nine selected for full screening. The study selection process, described using a preferred reporting items for systematic reviews and meta-analyses flow diagram ${ }^{(16)}$, is displayed in Fig. 1. Finally, nine RCT performed between 2007 and 2017 were included in the meta-analysis.

The characteristics of studies included in the review ${ }^{(17-25)}$ are summarised in online Supplementary Table S1. All studies were prospective, double blinded RCT, except for one study that was open labelled $^{(24)}$. The treatment group received a variety of doses of intravenous Fe, depending on baseline ferritin, with two studies using oral Fe preparations ${ }^{(20,25)}$. The control group received a placebo or no treatment at all. It should be noted that in the study of Beck-da-Silva et al. ${ }^{(19)}$ there were two treatment groups, intravenous Fe and oral Fe preparation. Only safety and tolerability of intravenous Fe preparation were reported as outcomes in Arutyunov et al.'s study ${ }^{(18)}$. A total of 1374 patients with chronic HF with reduced EF and ID were included, of whom 789 were included in the treatment arm and 585 in the control group.

\section{Risk of bias assessment}

Two researchers evaluated the risk of bias of the studies included in the review, according to the Cochrane risk of bias assessment tool $^{(14)}$. Online Supplementary Table S2 summarises the risk of bias results. No sufficient data were available to assess the risk of bias in the studies of Arutyunov et al. ${ }^{(18)}$ and Suryani et al. ${ }^{(25)}$.

\section{Outcomes}

Peak $\mathrm{VO}_{2}$. Three studies reported peak $\mathrm{VO}_{2} / \mathrm{kg}$ at the final follow-up. The studies were heterogeneous $\left(I^{2}=87 \%\right)$ and so a 


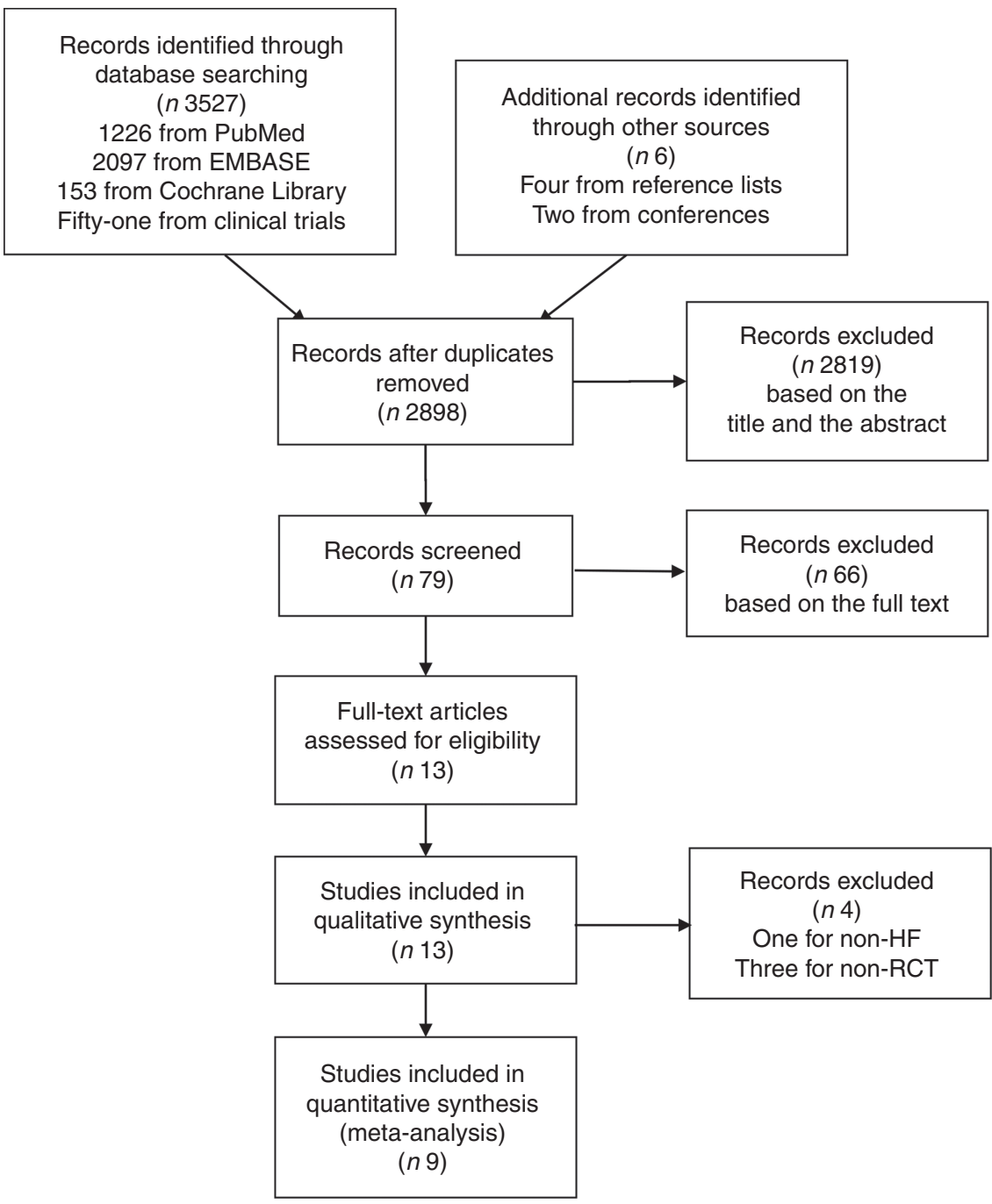

Fig. 1. Process of literature search and study selection. HF, heart failure; RCT, randomised control trial.

random effects model was used in the analysis, which demonstrated that Fe supplementation in the treatment group was associated with greater benefits to the peak $\mathrm{VO}_{2} / \mathrm{kg}$ in patients (Fig. 2(a)). Interestingly, this improvement was only observed in studies of intravenous Fe preparations, while oral Fe supplementation did not improve patient exercise tolerance.

\section{6-min walk test}

Five trials examined the effect of Fe on the 6MWT. The study published by Lewis et al. ${ }^{(20)}$ demonstrated that at the end of the 16-week follow-up, oral Fe supplementation had not increased the distance walked by patients in 6-min compared with baseline. The other four studies indicated that Fe supplementation (three intravenous and one oral) increased the 6-min walking distance compared with baseline. The overall heterogeneity was large $\left(I^{2}=91 \%\right)$ and analysis using a random effects model demonstrated that Fe supplementation failed to increase the distance of the 6MWT. Only when the Lewis et al. ${ }^{(20)}$ and Suryani et $a l .{ }^{(25)}$ studies were removed, the heterogeneity reduced significantly $\left(I^{2}=31 \%\right)$, and analysis using a fixed effects model demonstrated a significant increase in the distance of the 6MWT (Fig. 2(b)).

\section{All-cause mortality and cardiovascular death}

Seven studies reported the effect of Fe supplementation on cardiovascular death and all-cause mortality at the end of follow-up, with no apparent heterogeneity. Analysis by fixed effects model demonstrated that Fe supplementation failed to reduce all-cause mortality (OR: 0.70; 95\% CI 0.38, 1.28) (Fig. 3(a)). Three studies reported cardiovascular-related deaths with no apparent heterogeneity. Fixed-effects model analysis indicated that Fe supplementation did not reduce cardiovascular death in patients with chronic HF (OR: 0.81; $95 \%$ CI 0.40, 1.64) (Fig. 3(b)).

\section{Hospitalisation}

Five studies reported re-admission rate throughout the respective studies, indicating that intravenous Fe supplementation did not reduce the overall re-admission rate. Subgroup analyses were performed which indicated a significant decline 
(a)

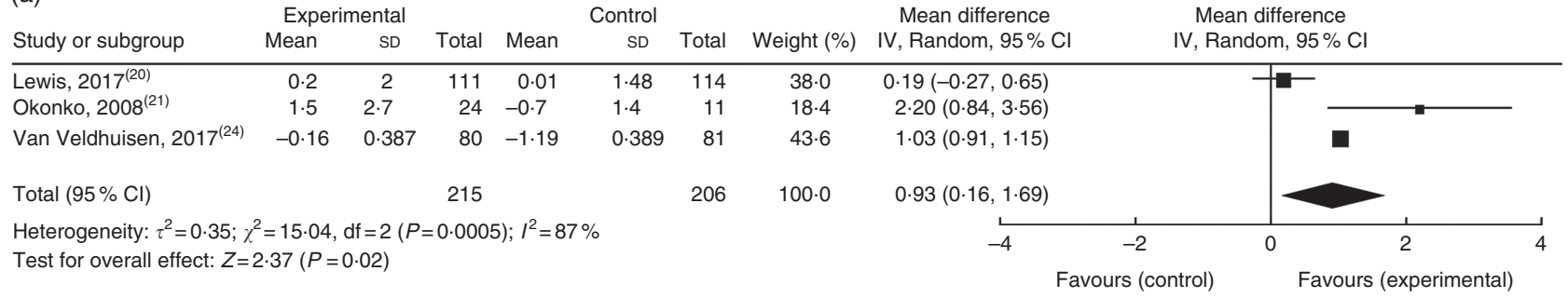

(b)

\begin{tabular}{|c|c|c|c|c|c|c|c|c|}
\hline & & e & & & Control & & & Mean difference \\
\hline Study or subgroup & Mean & SD & Total & Mean & SD & Total & Weight (\%) & IV, Random, $95 \% \mathrm{Cl}$ \\
\hline $2 \cdot 1 \cdot 1$ I.V. $\mathrm{Fe}$ & & & & & & & & \\
\hline Anker, $2009^{(17)}$ & 39 & $73 \cdot 4$ & 303 & 8 & $75 \cdot 7$ & 155 & $21 \cdot 0$ & $31.00(16 \cdot 50,45 \cdot 50$ \\
\hline Ponikowski, 2015 & 18 & $95 \cdot 6661$ & 143 & -16 & $97 \cdot 3242$ & 148 & $19 \cdot 5$ & $34.00(11.83,56 \cdot 17$ \\
\hline Toblli, $2007^{(23)}$ & $47 \cdot 8$ & $36 \cdot 6$ & 20 & $-6 \cdot 2$ & $36 \cdot 3$ & 20 & $19 \cdot 4$ & $54.00(31.41,76.59$ \\
\hline Subtotal $(95 \% \mathrm{CI})$ & & & 466 & & & 323 & $59 \cdot 8$ & $37 \cdot 84(24 \cdot 47,51 \cdot 22$ \\
\hline
\end{tabular}

Heterogeneity: $\tau^{2}=44 \cdot 72 ; \chi^{2}=2 \cdot 90, \mathrm{df}=2(P=0.23) ; I^{2}=31 \%$

Test for overall effect: $Z=5.55(P<0.00001)$

2.1.2 Oral Fe

Suryani, $2017^{(25)}$

$\begin{array}{llll}19 & 51.89 & 111 & 32\end{array}$

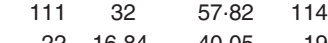

$40.05 \quad 19$ 133

$21 \cdot 0$

$19 \cdot 2$

$40 \cdot 2$

$-13.00(-27.35,1.35)$ $63.07(39.62,86.52)$ $24.45(-50.09,98.98)$

Heterogeneity: $\tau^{2}=2794.98 ; \chi^{2}=29.42, \mathrm{df}=1(P<0.00001) ; I^{2}=97 \%$

Test for overall effect: $Z=0.64(P=0.52)$

Total $(95 \% \mathrm{Cl})$

599

456

$100 \cdot 0$

Heterogeneity: $\tau^{2}=904.90 ; \chi^{2}=44.66, \mathrm{df}=4(P<0.00001) ; I^{2}=91 \%$

Test for overall effect: $Z=2.32(P=0.02)$

Test for subgroup differences: $\tau^{2}=0 \cdot 12 ; \mathrm{df}=1(P=0.73) ; I^{2}=0 \%$

Fig. 2. Effect of exercise tolerance on: (a) peak $\mathrm{VO}_{2}$ ( $\mathrm{ml} / \mathrm{kg}$ per $\left.\mathrm{min}\right)$; (b) sub-analysis of change in 6-min walk test distance compared with baseline.

in re-admission for HF (OR: 0.42; $95 \%$ CI 0.27, 0.65) (Fig. 3(c) and (d)).

\section{Serious adverse events}

Six studies reported serious adverse reactions, with sixty-four patients in the treatment group and seventy-four patients in the control group having at least one serious adverse event. Analysis using a fixed effects model demonstrated that the treatment group exhibited a lower incidence of serious adverse reactions (OR: 0.58; $95 \%$ CI 0.40, 0.83) (Fig. 4). No significant differences were observed in the adverse events, such as gastrointestinal dysfunction, nervous system adverse reaction or changes in skin mucosa.

\section{Quality-of-life parameters}

Two trials reported the MLHFQ score, with their pooled results demonstrating a statistical improvement (-19.47; $95 \%$ CI -23.36, -15.59). EQ-5D score was assessed by Anker et al. ${ }^{(17)}$ and Ponikowski et al. ${ }^{(22)}$. Compared with the control group, the Fe therapy group had a higher score, indicating improved clinical status (online Supplementary Fig. S1).

\section{Iron metabolism markers}

Six trials ${ }^{(17,20,21,23,24,25)}$ examined the effects of Fe supplementation on the level of Fe metabolism-related markers. Available Fe metabolic markers were TSAT, $\mathrm{Hb}$ and ferritin. The trials included in this review indicated that Fe supplementation increased ferritin and TSAT except the study published by Lewis et $a l^{(20)}$. Okonko et $a l .{ }^{(21)}$ suggested that Fe therapy improved ferritin and TSAT but did not increase $\mathrm{Hb}$ at the end of follow-up.

\section{Cardiac and renal function}

Four studies ${ }^{(17,21-23)}$ reported the effects of Fe supplementation on cardiac function. These findings all demonstrated an improvement or a tendency of improvement in cardiac function at the end of follow-up, in addition to an improvement in QoL. There was no report of improvement in cardiac function through the use of oral $\mathrm{Fe}$ supplementation. Five studies $^{(17,19-21,23)}$ reported the effects of Fe supplementation on renal function. Toblli et al. ${ }^{(23)}$ and Anker et al. ${ }^{(17)}$ demonstrated that there was a significant improvement in renal function. Okonko et al. ${ }^{(21)}$, Lewis et al. ${ }^{(20)}$ and Beck-da-Silva et al. ${ }^{(19)}$ found no change in renal function over the duration of the respective studies. No decline in renal function was observed in any of the studies.

\section{Sensitivity analysis}

The analysis above demonstrated that heterogeneity was significantly reduced when the studies published by Lewis et al. ${ }^{(20)}$ and Suryani et $a l .{ }^{(25)}$, which analysed 6MWT distance and peak $\mathrm{VO}_{2} / \mathrm{kg}$ compared with the baseline, were removed. Eliminating the trials individually from the analysis had no relevant effect on the overall outcome. Recalculating the analysis using a 
(a)

\begin{tabular}{|c|c|c|c|c|c|c|c|c|c|}
\hline \multirow[b]{2}{*}{ Study or subgroup } & \multicolumn{2}{|c|}{ Experimental } & \multicolumn{2}{|c|}{ Control } & \multirow{2}{*}{$\begin{array}{l}\text { Weight } \\
(\%)\end{array}$} & \multirow{2}{*}{$\begin{array}{c}\text { OR } \\
\mathrm{M}-\mathrm{H}, \text { Fixed, } 95 \% \mathrm{Cl}\end{array}$} & \multirow{2}{*}{\multicolumn{2}{|c|}{$\begin{array}{c}\text { OR } \\
\mathrm{M}-\mathrm{H}, \text { Fixed, } 95 \% \mathrm{Cl}\end{array}$}} & \\
\hline & Events & Total & Events & Total & & & & & \\
\hline Anker, $2009^{(17)}$ & 5 & 305 & 4 & 154 & $20 \cdot 9$ & $0 \cdot 63(0 \cdot 17,2 \cdot 36)$ & $\longrightarrow$ & - & \\
\hline Arutyunov, $2009^{(18)}$ & 1 & 57 & 0 & 15 & $3 \cdot 1$ & $0.82(0.03,21.22)$ & & & \\
\hline Beck-da-Silva, 2013 ${ }^{(19)}$ & 2 & 10 & 1 & 6 & $4 \cdot 0$ & $1.25(0.09,17.65)$ & & & \\
\hline Okonko, $2008^{(21)}$ & 1 & 24 & 0 & 11 & 2.5 & $1.47(0.06,38.91)$ & & & \\
\hline Ponikowski, 2015(22) & 12 & 150 & 14 & 151 & $51 \cdot 4$ & $0.85(0.38,1.91)$ & - & & \\
\hline Van Veldhuisen, 2017 (24) & 0 & 88 & 4 & 86 & $18 \cdot 1$ & $0.10(0.01,1.95)$ & & - & \\
\hline Total $(95 \% \mathrm{Cl})$ & & 634 & & 423 & $100 \cdot 0$ & $0 \cdot 70(0 \cdot 38,1 \cdot 28)$ & & & \\
\hline Total events & 21 & & 23 & & & & & & \\
\hline \multirow{2}{*}{\multicolumn{5}{|c|}{$\begin{array}{l}\text { Heterogeneity: } \chi^{2}=2 \cdot 27, \mathrm{df}=5(P=0.81) ; I^{2}=0 \% \\
\text { Test for overall effect: } Z=1 \cdot 16(P=0.25)\end{array}$}} & & 0.005 & 0.1 & 10 & 200 \\
\hline & & & & & & & (experimental) & Favours (c & \\
\hline
\end{tabular}

(b)

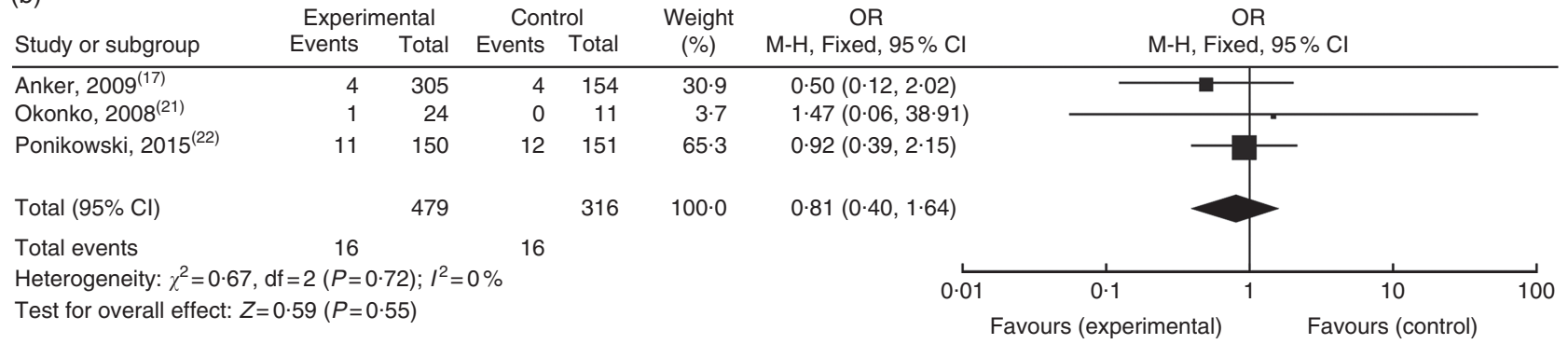

(c)

\begin{tabular}{|c|c|c|c|c|c|c|c|c|c|c|}
\hline \multirow[b]{2}{*}{ Study or subgroup } & \multicolumn{2}{|c|}{ Experimental } & \multicolumn{2}{|c|}{ Control } & \multirow{2}{*}{$\begin{array}{l}\text { Weight } \\
(\%)\end{array}$} & \multirow{2}{*}{$\stackrel{\text { OR }}{\mathrm{M}-\mathrm{H}, \text { Random, } 95 \% \mathrm{Cl}}$} & \multirow{2}{*}{\multicolumn{3}{|c|}{$\begin{array}{c}\text { OR } \\
\mathrm{M}-\mathrm{H}, \text { Random, } 95 \% \mathrm{Cl}\end{array}$}} & \\
\hline & Events & Total & Events & Total & & & & & & \\
\hline Anker, 2009 $9^{(17)}$ & 28 & 305 & 22 & 154 & $27 \cdot 3$ & $0.61(0.33,1 \cdot 10)$ & & $\Longrightarrow$ & & \\
\hline Okonko, $2008^{(21)}$ & 3 & 20 & 3 & 10 & $11 \cdot 6$ & $0.41(0.07,2.56)$ & & & & \\
\hline Ponikowski, $2015^{(22)}$ & 46 & 150 & 69 & 151 & $29 \cdot 0$ & $0.53(0.33,0.84)$ & & - & & \\
\hline Toblli, $2007^{(23)}$ & 0 & 20 & 5 & 20 & $5 \cdot 7$ & $0.07(0.00,1.34)$ & & & & \\
\hline Van Veldhuisen, $2017^{(24)}$ & 37 & 88 & 21 & 86 & $26 \cdot 5$ & $2 \cdot 25(1 \cdot 17,4 \cdot 30)$ & & & $\longrightarrow$ & \\
\hline Total $(95 \% \mathrm{Cl})$ & & 583 & & 421 & $100 \cdot 0$ & $0.70(0.32,1 \cdot 51)$ & & & & \\
\hline Total events & 114 & & 120 & & & & & & & \\
\hline \multicolumn{7}{|c|}{ Heterogeneity: $\tau^{2}=0.48 ; \chi^{2}=16.54, \mathrm{df}=4(P=0.002) ; I^{2}=76 \%$} & 0.05 & 0.2 & 1 & 5 \\
\hline \multicolumn{7}{|c|}{ Test for overall effect: $Z=0.92(P=0.36)$} & Favo & (experimental) & Favours & s (control) \\
\hline
\end{tabular}

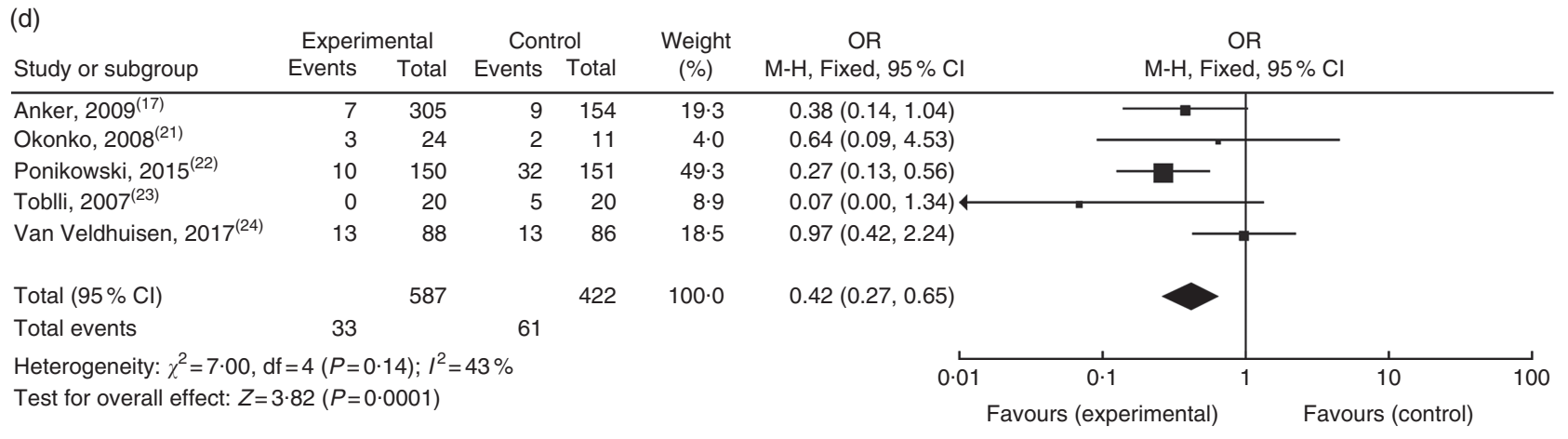

Fig. 3. Effect on: (a) all causes of death; (b) cardiovascular death; (c) re-hospitalisation and (d) re-hospitalisation for heart failure at the end of follow-up.

random effects model instead of a fixed effects model did not change the overall outcome.

\section{Discussion}

In the present meta-analysis, we included available data on the treatment effect and safety of Fe supplementation in patients with HFrEF having ID. The analysis demonstrated that Fe supplementation increased peak $\mathrm{VO}_{2} / \mathrm{kg}$ and reduced re-admission for $\mathrm{HF}$ without increasing the incidence of serious adverse events but failed to reduce mortality. Unlike intravenous supplementation, oral Fe supplementation increased transferrin concentration and serum Fe levels but did not improve peak oxygen uptake, 6-min walking distance, N-terminal pro-hormone of brain natriuretic peptide, Kansas City 


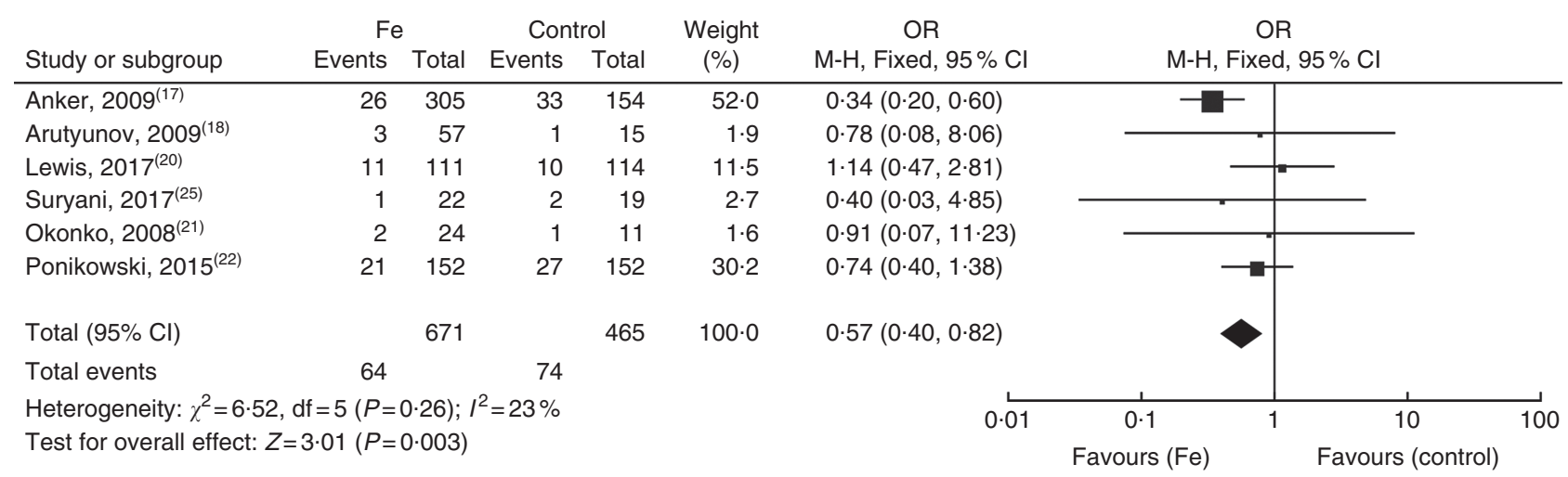

Fig. 4. Forest plot showing the serious adverse effects.

Cardiomyopathy Questionnaire scores and failed to reduce HF re-admission or rates of death.

HF patients often have many co-morbidities that worsen their $\operatorname{prognosis}^{(26,27)}$. Currently, recommendations for HF therapy include $\beta$-blockers, angiotensin-converting enzyme inhibitors, aldosterone receptor antagonists and diuretics, which perform a critical role in the management of $\mathrm{HF}^{(28,29)}$. However, despite the standard-of-care regimens, the mortality and re-admission rates for $\mathrm{HF}$ are still high, suggesting that the overall treatment strategy for patients with HF should consider other co-morbidities ${ }^{(30)}$.

Fe plays a crucial role in many biological functions, including energy production, cell proliferation, oxygen delivery and storage (as a component of myoglobin) and oxidative metabolism in skeletal and heart muscles. Fe is an obligate element of $\mathrm{Hb}$ and many types of enzymes, participating in various cellular processes. ID may be detrimental to the cardiovascular system ${ }^{(31)}$. Maeder et al. $^{(32)}$ demonstrated that both Fe content in tissues and type 1 transferrin receptor expression in HF patients were reduced. Myoglobin is the primary oxygen-carrying and binding protein found in muscles. Mitochondrial function also requires $\mathrm{Fe}$ since it is a co-factor for many proteins involved in electron transfer and in ATP and energy production in cells.

ID describes a diminished concentration of $\mathrm{Fe}$ in stores and hence cannot meet the eventual demand. ID is among the most prevalent co-morbid condition in patients with CHD and chronic $\mathrm{HF}^{(6,33)}$. Exercise tolerance is affected due to reduced oxygen storage in myoglobin, reduced energy efficiency and mitochondrial dysfunction when $\mathrm{Fe}$ storage is depleted ${ }^{(34)}$. Several observational studies have demonstrated that ID is associated with an increased mortality rate in CHF. Jankowska et $a l{ }^{(35)}$ found that ID was a poor prognostic factor in many patients with $\mathrm{CHF}$, including hospitalisation and death. The 3year survival rate was $59 \%$ in patients with ID and $71 \%$ for patients without ID $(P=0.0006)$.

A previous meta-analysis (Avni et al. ${ }^{(36)}$ ) also showed that Fe repletion was associated with improved QoL parameters and safety for $\mathrm{HF}$ patients. Our study further confirmed that $\mathrm{Fe}$ supplementation can improve peak oxygen uptake and readmission in patients with HFrEF, which was not reported in previous meta-analysis studies.

Lewis et al. ${ }^{(20)}$ published the first multicentre randomised controlled clinical trial exploring the effects and safety of oral Fe supplementation in patients with HFrEF having $\mathrm{ID}^{(20)}$. There are several explanations for the failure of oral Fe supplementation to improve exercise tolerance in patients with HFrEF. The absorption of Fe from oral preparations is generally poor and, furthermore, up to $60 \%$ of patients experience gastrointestinal side effects after its administration ${ }^{(37)}$. The absorption of Fe from the gastrointestinal tract may be limited by numerous foods and drugs, and intestinal mucosal oedema caused by systemic venous congestion in $\mathrm{HF}$ patients also results in a decrease in $\mathrm{Fe}$ absorption $^{(38)}$. Hepcidin is a peptide hormone secreted by the liver that regulates Fe homoeostasis ${ }^{(39)}$. Hepcidin modulates Fe release by causing the degradation of Fe transporters ${ }^{(40,41)}$. In a recent study by Jankowska et al. ${ }^{(42)}$, systolic HF appeared to be the result of high levels of circulating hepcidin, which caused unnaturally high rates of Fe transporter degradation.

A variety of Fe complexes were assessed in the trials in this review, such as ferric carboxymaltose, Fe sucrose, etc. Although there is limited comparative data for IV Fe complexes, this review establishes that Fe supports improvement in many parameters in patients with HFrEF having ID.

In the sensitivity analysis, eliminating the study published by van Veldhuisen et $a l .{ }^{(24)}$ resulted in more apparent differences in total re-hospitalisation rates during the follow-up period, which may be due to non-cardiovascular reasons. This exclusion did not change the trend in re-hospitalisation following an analysis related to HF. After removing the study published by Lewis et al. ${ }^{(20)}$, Fe therapy resulted in a much greater increase in peak $\mathrm{VO}_{2} / \mathrm{kg}$, while the numbers of serious adverse reactions did not change, also suggesting that oral Fe supplementation failed to improve the peak $\mathrm{VO}_{2}$, though not increasing the number of adverse reactions. The reasons mentioned above are likely to be the cause of this heterogeneity.

This review found that both oral and intravenous Fe supplementation did not increase the incidence of adverse reactions, such as gastrointestinal and nervous system disorders, and conversely, adverse effects in the treatment group were lower than that in the control group (OR: 0.58; 95\% CI 0.40, $0 \cdot 83$ ), suggesting the safety of Fe supplementation. Furthermore, Fe supplementation could even reduce the symptoms in patients with ID and HF.

There are some limitations in this review, that is, the inclusion of an open-label study and those of oral Fe supplementation, 
which may be sources of heterogeneity. In addition, total $\mathrm{Fe}$ dose varied, as did the duration of follow-up, which ranged from 16 to 52 weeks, so generalisation of the findings should be done with caution.

Despite these limitations, this meta-analysis suggests that patients with HFrEF having ID may benefit from Fe supplementation without increasing the incidence of adverse events. Additionally, a larger sample size, longer follow-up and multicentre prospective clinical studies are required to confirm whether Fe supplementation can reduce mortality and improve long-term prognosis. Whether oral Fe supplementation can improve the exercise tolerance of patients with HF and prognosis requires additional persuasive research.

The findings of this meta-analysis of RCT suggest that intravenous but not oral Fe supplementation can increase the peak $\mathrm{VO}_{2} / \mathrm{kg}$ and 6MWT and reduce re-admission for HF, without increasing the incidence of serious adverse events. This may provide a possible therapeutic target for patients with HFrEF having ID. Future clinical studies with larger sample sizes that focus on mortality are required.

\section{Acknowledgements}

This review was supported by the National Natural Science Foundation of China (no. 81400303). The funder had no role in the design or conduct of the study; collection, management, analysis or interpretation of the data; preparation, review or approval of the manuscript; or the decision to submit the manuscript for publication.

C. W., S. Z. and F. Z. designed the study; S. Z., M. D. and K. H. collected the data; F. Z. performed the statistical analysis and drafted the manuscript; C. W., S. Z., F. Z., M. D. and K. H. critically revised the manuscript for important intellectual content and $\mathrm{C}$. W. supervised the work and had primary responsibility for the final content. All authors read and approved the final manuscript. S. Z. and F. Z. did the literature search, screened the results, extracted and analysed data from included trials. M. D. and K. H. helped perform the analysis with constructive discussions. C. W. designed the study, performed the research, analysed data and wrote the paper. This author takes responsibility for all aspects of the reliability and freedom from bias of the data presented and their discussed interpretation.

The authors declare that there are no conflicts of interest.

\section{Supplementary material}

For supplementary material/s referred to in this article, please visit https://doi.org/10.1017/S000711451900014X

\section{References}

1. Stewart S, Jenkins A, Buchan S, et al. (2002) The current cost of heart failure to the National Health Service in the UK. Eur J Heart Fail 4, 361-371.

2. Cook C, Cole G, Asaria P, et al. (2014) The annual global economic burden of heart failure. Int J Cardiol 171, 368-376.

3. Weiwei C, Runlin G, Lisheng L, et al. (2016) Outline of the report on cardiovascular diseases in China, 2014. Eur Heart J Suppl 18, F2-F11.
4. Writing Group Members, Mozaffarian D, Benjamin EJ, et al. (2016) Heart disease and stroke statistics-2016 update: a report from the American Heart Association. Circulation 133, e38-e360.

5. Roger VL (2013) Epidemiology of heart failure. Circ Res $\mathbf{1 1 3}$, 646-659.

6. Jankowska EA, von Haehling S, Anker SD, et al. (2013) Iron deficiency and heart failure: diagnostic dilemmas and therapeutic perspectives. Eur Heart J 34, 816-829.

7. Buratti P, Gammella E, Rybinska I, et al. (2015) Recent advances in iron metabolism: relevance for health, exercise, and performance. Med Sci Sports Exerc 47, 1596-1604.

8. Cohen-Solal A, Leclercq C, Deray G, et al. (2014) Iron deficiency: an emerging therapeutic target in heart failure. Heart 100, 1414-1420.

9. Wong CC, Ng AC, Kritharides L, et al. (2016) Iron deficiency in heart failure: looking beyond anaemia. Heart Lung Circ 25, 209-216.

10. Klip IT, Comin-Colet J, Voors AA, et al. (2013) Iron deficiency in chronic heart failure: an international pooled analysis. $\mathrm{Am}$ Heart J 165, 575-582.e573.

11. Silverberg DS, Wexler D, Sheps D, et al. (2001) The effect of correction of mild anemia in severe, resistant congestive heart failure using subcutaneous erythropoietin and intravenous iron: a randomized controlled study. J Am Coll Cardiol 37, $1775-1780$

12. Bolger AP, Bartlett FR, Penston HS, et al. (2006) Intravenous iron alone for the treatment of anemia in patients with chronic heart failure. J Am Coll Cardiol 48, 1225-1227.

13. Higgins JPT \& Green S (2011) Cochrane handbook for systematic reviews of interventions version 5.1.0 (updated March 2011). The Cochrane Collaboration. http://handbook.cochrane.org (accessed January 2018).

14. Higgins JP, Altman DG, Gotzsche PC, et al. (2011) The Cochrane Collaboration's tool for assessing risk of bias in randomised trials. BMJ 343, d5928.

15. Hozo SP, Djulbegovic B \& Hozo I (2005) Estimating the mean and variance from the median, range, and the size of a sample. BMC Med Res Methodol 5, 13.

16. Moher D, Liberati A, Tetzlaff J, et al. (2009) Preferred reporting items for systematic reviews and meta-analyses: the PRISMA statement. BMJ 339, b2535.

17. Anker SD, Comin Colet J, Filippatos G, et al. (2009) Ferric carboxymaltose in patients with heart failure and iron deficiency. N Engl J Med 361, 2436-2448.

18. Arutyunov GP, Bylova NA, Ivleva AY, et al. (2009) The safety of intravenous (IV) ferric carboxymaltose $v$. IV iron sucrose in patients with chronic heart failure (CHF) and chronic kidney disease (CKD) with iron deficiency (ID). Eur J Heart Fail Suppl 8, ii71-ii71, Abstract 141.

19. Beck-da-Silva L, Piardi D, Soder S, et al. (2013) IRON-HF study: a randomized trial to assess the effects of iron in heart failure patients with anemia. Int J Cardiol 168, 3439-3442.

20. Lewis GD, Malhotra R, Hernandez AF, et al. (2017) Effect of oral iron repletion on exercise capacity in patients with heart failure with reduced ejection fraction and iron deficiency: the IRONOUT HF randomized clinical trial. JAMA 317, 1958-1966.

21. Okonko DO, Grzeslo A, Witkowski T, et al. (2008) Effect of intravenous iron sucrose on exercise tolerance in anemic and nonanemic patients with symptomatic chronic heart failure and iron deficiency FERRIC-HF: a randomized, controlled, observer-blinded trial. J Am Coll Cardiol 51, 103-112.

22. Ponikowski P, van Veldhuisen DJ, Comin-Colet J, et al. (2015) Beneficial effects of long-term intravenous iron therapy with ferric carboxymaltose in patients with symptomatic heart failure and iron deficiency. Eur Heart J 36, 657-668. 
23. Toblli JE, Lombraña A, Duarte $\mathrm{P}$, et al. (2007) Intravenous iron reduces NT-Pro-brain natriuretic peptide in anemic patients with chronic heart failure and renal insufficiency. J Am Coll Cardiol 50, 1657-1665.

24. van Veldhuisen DJ, Ponikowski $\mathrm{P}$, van $\operatorname{der}$ Meer $\mathrm{P}$, et al. (2017) Effect of ferric carboxymaltose on exercise capacity in patients with chronic heart failure and iron deficiency. Circulation 136, 1374-1383

25. Suryani LD, Raharjo SB, Sagita R, et al. (2017) Oral iron therapy improves functional capacity of heart failure patients with iron deficiency anaemia. Eur Heart J Suppl 19, E17-E17.

26. Ambrosy AP, Fonarow GC, Butler J, et al. (2014) The global health and economic burden of hospitalizations for heart failure: lessons learned from hospitalized heart failure registries. J Am Coll Cardiol 63, 1123-1133.

27. Gheorghiade M \& Pang PS (2009) Acute heart failure syndromes. J Am Coll Cardiol 53, 557-573.

28. Yancy CW, Jessup M, Bozkurt B, et al. (2017) 2017 ACC/AHA/ HFSA focused update of the $2013 \mathrm{ACCF} / \mathrm{AHA}$ guideline for the management of heart failure. A report of the American College of Cardiology/American Heart Association Task Force on Clinical Practice Guidelines and the Heart Failure Society of America. Circulation 136, e137-e161.

29. Ponikowski P, Voors AA, Anker SD, et al. (2016) 2016 ESC guidelines for the diagnosis and treatment of acute and chronic heart failure: the task force for the diagnosis and treatment of acute and chronic heart failure of the European Society of Cardiology (ESC) developed with the special contribution of the Heart Failure Association (HFA) of the ESC. Eur Heart J 37, 2129-2200.

30. van Deursen VM, Urso R, Laroche C, et al. (2014) Comorbidities in patients with heart failure: an analysis of the European Heart Failure Pilot Survey. Eur J Heart Fail 16, 103-111.
31. von Haehling S, Jankowska EA, van Veldhuisen DJ, et al. (2015) Iron deficiency and cardiovascular disease. Nat Rev Cardiol 12, 659-669.

32. Maeder MT, Khammy O, dos Remedios C, et al. (2011) Myocardial and systemic iron depletion in heart failure implications for anemia accompanying heart failure. $\mathrm{J} \mathrm{Am}$ Coll Cardiol 58, 474-480.

33. Ebner N \& von Haehling S (2013) Iron deficiency in heart failure: a practical guide. Nutrients 5, 3730-3739.

34. Haas JD \& Brownlie TT (2001) Iron deficiency and reduced work capacity: a critical review of the research to determine a causal relationship. J Nutr 131, 676S-688S; discussion 688S-690S.

35. Jankowska EA, Rozentryt P, Witkowska A, et al. (2010) Iron deficiency: an ominous sign in patients with systolic chronic heart failure. Eur Heart J 31, 1872-1880.

36. Avni T, Leibovici L \& Gafter-Gvili A (2012) Iron supplementation for the treatment of chronic heart failure and iron deficiency: systematic review and meta-analysis. Eur J Heart Fail 14, 423-429.

37. Anker SD \& von Haehling S (2012) Iron deficiency and anemia in heart failure. Chapter 3, 26-35.

38. Sandek A, Bjarnason I, Volk HD, et al. (2012) Studies on bacterial endotoxin and intestinal absorption function in patients with chronic heart failure. Int J Cardiol 157, 80-85.

39. Ohno Y, Hanawa H, Jiao S, et al. (2015) Liver congestion in heart failure contributes to inappropriately increased serum hepcidin despite anemia. Tohoku J Exp Med 235, 69-79.

40. Franchini M, Montagnana M \& Lippi G (2010) Hepcidin and iron metabolism: from laboratory to clinical implications. Clin Chim Acta 411, 1565-1569.

41. Ganz T (2011) Hepcidin and iron regulation, 10 years later. Blood 117, 4425-4433.

42. Jankowska EA, Malyszko J, Ardehali H, et al. (2013) Iron status in patients with chronic heart failure. Eur Heart J34, 827-834. 